\title{
How Does Quality of Life Relate to Auditory Abilities? A Subitem Analysis of the Nijmegen Cochlear Implant Questionnaire
}

\author{
Kara J. Vasil ${ }^{1}$ Jessica Lewis ${ }^{1}$ Terrin Tamati ${ }^{1}$ Christin Ray ${ }^{1}$ Aaron C. Moberly ${ }^{1}$ \\ 1 Department of Otolaryngology-Head \& Neck Surgery, The Ohio \\ State University, Columbus, $\mathrm{OH}$ \\ Address for correspondence Kara J. Vasil, Department of \\ Otolaryngology-Head \& Neck Surgery, The Ohio State University \\ J Am Acad Audiol 2020;31:292-301. \\ Wexner Medical Center, $\mathrm{OH}$ 43212, Columbus \\ (e-mail: Kara.Vasil@osumc.edu).
}

\begin{abstract}
Keywords

- cochlear implants

- environmental sound recognition

- patient-reported outcome measures

- quality of life

- speech recognition
\end{abstract}

Background Objective speech recognition tasks are widely used to measure performance of adult cochlear implant $(\mathrm{Cl})$ users; however, the relationship of these measures with patient-reported quality of life $(\mathrm{QOL})$ remains unclear. A comprehensive QOL measure, the Nijmegen Cochlear Implant Questionnaire (NCIQ), has historically shown a weak association with speech recognition performance, but closer examination may indicate stronger relations between $\mathrm{QOL}$ and objective auditory performance, particularly when examining a broad range of auditory skills.

Purpose The aim of the present study was to assess the NCIQ for relations to speech and environmental sound recognition measures. Identifying associations with certain QOL domains, subdomains, and subitems would provide evidence that speech and environmental sound recognition measures are relevant to QOL. A lack of relations among QOL and various auditory abilities would suggest potential areas of patientreported difficulty that could be better measured or targeted.

Research Design A cross-sectional study was performed in adult $\mathrm{Cl}$ users to examine relations among subjective $\mathrm{QOL}$ ratings on $\mathrm{NCIQ}$ domains, subdomains, and subitems with auditory outcome measures.

Study Sample Participants were 44 adult experienced $\mathrm{Cl}$ users. All participants were postlingually deafened and had met candidacy requirements for traditional cochlear implantation.

Data Collection and Analysis Participants completed the NCIQ as well as several speech and environmental sound recognition tasks: monosyllabic word recognition, standard and high-variability sentence recognition, audiovisual sentence recognition, and environmental sound identification. Bivariate correlation analyses were performed to investigate relations among patient-reported NCIQ scores and the functional auditory measures.

Results The total NCIQ score was not strongly correlated with any objective auditory outcome measures. The physical domain and the advanced sound perception subdomain related to several measures, in particular monosyllabic word recognition and AzBio sentence recognition. Fourteen of the 60 subitems on the NCIQ were correlated with at least one auditory measure.

Conclusions Several subitems demonstrated moderate-to-strong correlations with auditory measures, indicating that these auditory measures are relevant to the QOL. A
Copyright $\odot 2020$ by the American Academy of Audiology. All rights reserved. Thieme Medical Publishers, Inc., 333 Seventh Avenue, New York, NY 10001, USA. Tel: +1(212) 760-0888
DOI https://doi.org/ 10.3766/jaaa.19047. ISSN 1050-0545. 
lack of relations with other subitems suggests a need for the development of objective measures that will better capture patients' hearing-related obstacles. Clinicians may use information obtained through the NCIQ to better estimate real-world performance, which may support improved counseling and development of recommendations for $\mathrm{Cl}$ patients.

For adults with severe-to-profound hearing loss, cochlear implants (CIs) are the proven standard of care. To assess functional outcomes, patients are typically evaluated using objective, open-set speech recognition tests, most commonly in quiet. Most studies confirm dramatic improvements in speech recognition performance after cochlear implantation (Gaylor et al, 2013 ${ }^{7}$ ).

However, these objective speech recognition measures have been criticized as not fully capturing the benefits that the $\mathrm{CI}$ has for the recipient, and hearing-related quality of life (QOL) and other patient-reported outcome (PRO) self-assessment measures have been found to be useful supplements. Some of these PRO measures have included the Nijmegen Cochlear Implant Questionnaire (NCIQ) (Hinderink et al, $2000^{9}$ ), the Hearing Handicap Inventory for Adults (Newman et al, $1990^{17}$ ), and the Speech, Spatial, and Qualities of Hearing Scale (Gatehouse and Noble, $2004^{6}$ ) to assess patient's subjective hearing performance in several functional domains, such as physical, social, and emotional functions. Indeed, in recent years, federal organizations such as the Food and Drug Administration have been placing increased emphasis on PRO measures to "incorporate the patient perspective as evidence in our decisions" and assess the impact of disease and subsequent intervention on the patient's life (FDA-NIH Biomarker Working Group, $2016^{4}$ ). Hence, there is a need for clinical centers to adopt a CI-specific QOL measure to identify key issues from the patient's perspective and target these issues directly in clinical care. To accomplish this, the PRO measure must be comprehensive (pertaining to several aspects of the patient's life as they are affected by the hearing loss) and proven valid in the adult $\mathrm{CI}$ population. Specifically, the NCIQ is a disease-specific PRO measure ofQOL that has been found to be reliable and valid among CI users (Hinderink et al, 2000; ${ }^{9}$ Krabbe et al, 2000 ${ }^{9}$ ), and it assesses physical, psychological, and social facets of hearing loss. The assessment was constructed by using conventional domain criteria (physical, psychological, and social domains), and subitems were developed based on interviews with $\mathrm{CI}$ users as well as adapted items from other widely used questionnaires. Thus, the NCIQ holds promise as one of the more valuable presently available CI-specific PRO assessments of QOL.

Traditionally, objective speech recognition and subjective QOL measures do not show a strong relationship.

A meta-analysis by McRackan et al $(2018)^{14}$ indicated that of 14 articles examining QOL in adults with CIs, all studies demonstrated significant improvements in both QOL and speech recognition after implantation. However, standard speech recognition measures only showed small correlations $(r=0.20-0.26)$ with hearing-specific and CI-specific QOL measures.

To demonstrate effectiveness of CIs and their impact on patient's lives, it is important to thoroughly investigate the relationship of these subjective PRO measures with objective outcome measures, specifically speech recognition ability. Where objective and subjective measures do relate, an improved understanding of this relationship will provide a better sense of how our objective measures relate to patientreported changes in daily function as a result of $\mathrm{CI}$ intervention. Conversely, identifying where objective and subjective measures do not relate will reveal other, less frequently measured domains of patient performance and QOL, which are arguably equally as important in quantifying functional improvements. As demonstrated by McRackan et al $(2018)^{14}$, very few studies have examined more specific subdomain data in relation to objective measures, specifically speech recognition. Of the few studies which did examine subdomain data in analyses, a moderate correlation of the advanced speech perception subdomain of the NCIQ with word recognition in quiet was observed $\left(r=0.55\right.$ ) (Capretta and Moberly, 2016 ${ }^{2}$ ). Other subdomains indicated negligible or weak correlations with word recognition in quiet, sentence recognition in quiet, and sentence recognition in noise.

The present study aimed to further examine the relationships of CI-related QOL and objective measures by asking the following question: Where do our subjective QOL measures (including the domains, subdomains, and subitems from the NCIQ) overlap with CI user's auditory performance? Where there is overlap between subjective and objective measures, it would be evident that these outcome measures are relevant to patient-reported QOL; where there is not an association, we can identify areas of patient-reported difficulty for which we might develop measures to better quantify during audiologic testing. We predict that the objective auditory measures used in this study, which differ from most traditionally used clinical measures by incorporating audiovisual presentation, more linguistic complexity, and nonspeech testing, may better capture the everyday auditory experiences that CI users encounter. For example, individuals with hearing loss are known to rely on visual cues in conversation (Moberly et al, $2019^{16}$ ); therefore, an evaluation of audiovisual speech recognition (City University of New York [CUNY]) (Boothroyd et al, $1985^{1}$ ) should indicate a strong relationship with conversational ability as reported on a measure of QOL. In addition, more complex "Harvard Standard" sentences spoken by a single talker (IEEE, $1969^{11}$ ) and PRESTO high-talker-variability sentences (Gilbert et al, $2013^{8}$ ) were included in this battery to 
assess recognition ability for more challenging sentences spoken by multiple talkers with various regional dialects. Environmental sound recognition (as measured by the Familiar Environmental Sound Test [FEST-I]) (Shafiro, 2008 ${ }^{19}$ ) should relate to basic and advanced sound perception, two crucial subdomains included in the NCIQ (Hinderink et al, $2000^{9}$ ). In addition to these measures, clinical AzBio (Spahr et al, $2012^{20}$ ) scores were examined.

In this study, two main hypotheses were tested: first, we hypothesized that specific NCIQ domains, subdomains, and subitems relating to sound perception would be more strongly correlated with scores on our objective functional measures than the NCIQ total score. Second, we predicted that our expanded battery of objective auditory measures would demonstrate stronger correlations with NCIQ scores than found for previous auditory measures (e.g., Capretta and Moberly, 2016; ${ }^{2}$ McRackan et al, $2018^{14}$ ) because of our measure's increased complexity and their use of audiovisual and environmental sound stimuli, which should be more relevant to daily life as a $\mathrm{CI}$ user.

\section{Methods}

\section{Participants}

Participants were 44 experienced postlingually deafened $\mathrm{CI}$ users between the ages of 45 and 83 years. Selfreported duration of hearing loss ranged from 4 to 76 years, with the duration of $\mathrm{CI}$ use ranging from 18 months to 40 years. All participants were native English speakers with a high-school diploma or equivalency. They were screened for cognitive impairment using a written version of the Mini-Mental State Examination (Folstein et al, $1975^{5}$ ) and screened for basic word reading with the Wide Range Achievement Test (Wilkinson and Robertson, 2006 22 ). All participants demonstrated sufficient visual ability using a basic near-vision test to complete tests and questionnaires.

CI participants met traditional candidacy requirements before implantation and had at least 12 months of experience with their devices. Participants were recruited from a tertiary care adult Neurotology center. Of these, 13 participants (29.5\%) were bilaterally implanted. All CI users reported postlingual onset of deafness, with 32 (72.7\%) reporting onset of deafness after the age of 12 years. The other 12 (27.3\%) CI users reported some degree of hearing loss congenitally or during childhood. Of these participants, all had experienced intervention with amplification at an early age and used spoken English language as their primary form of communication. All CI users received their CIs at the age of 35 years or later. Thirteen participants were bilateral $\mathrm{CI}$ users (mean age 62.6 years, standard deviation [SD] 9.3; mean CI duration 8.7 years, SD 5.2); 18 participants were bimodal (i.e., using a hearing aid on their nonimplanted ear) (mean age 68.9 years, SD 10.8; mean CI duration 6.9 years, SD 9.0). Thirteen participants were unilateral CI users (mean age 68.1 years, SD 9.1; mean CI duration 5.4 years, SD 3.1). Twenty-five of the participants (56.8\%) from this sample were also included in a previous study of QOL as it relates to auditory perception and neurocognitive outcomes in adults with CIs (Moberly et al, $2018^{15}$ ), and minimal correlations were found between QOL and outcome measures used in that study. In the present study, we were able to expand on this knowledge with a larger sample size, additional outcome measures of increased difficulty and variability, and more specific subitem analyses.

\section{Equipment and Materials}

All testing took place within sound-proof booths and acoustically insulated testing rooms. Tests requiring verbal responses from participants were audiovisually recorded for later scoring, except for AzBio sentences, which were live-scored by a clinical audiologist.

Visual stimuli were presented on a computer monitor placed two feet in front of the participant. Laboratory auditory stimuli were presented via a Roland MA-12C (Roland Corporation, Hamamatsu, Japan) speaker placed $1 \mathrm{~m}$ in front of the participant at zero degrees azimuth. Before the testing session, the speaker was calibrated to $68 \mathrm{~dB}$ SPL using a sound-level meter. AzBio sentences were presented in the clinic at $60 \mathrm{~dB}$ SPL. All auditory and audiovisual measures were presented in quiet.

\section{Auditory and Audiovisual Measures}

One list of 12 CUNY sentences was administered (Boothroyd et al, $1985^{1}$ ) audiovisually. Sentence lists were randomized among participants. The sentences were spoken by a single female talker, and they varied in length and subject matter, for example, "Put snow tires on the car today." Participants were scored on the percentage of words repeated correctly.

Sentence recognition was also measured using 28 of the Harvard Standard sentences (IEEE, $1969^{11}$ ). These sentences are long, complex, and semantically meaningful, for example, "Glue the sheet to the dark blue background." A list of 30 highvariability PRESTO sentences (Gilbert et al, $2013^{8}$ ) was also presented; in this list, each sentence was spoken by a different male or female talker and included various regional dialects, like "Pam gives driving lessons on Thursdays." Word recognition was measured using Central Institute for the Deaf-W-22 (CIDW-22) word list (Hirsh et al, 1952 ${ }^{10}$ ). This list contained 50 monosyllabic words preceded by the carrier phrase, "Say the word." The CID W-22 word list was used in this study as previous studies have demonstrated a broad range of word recognition performance with minimal ceiling and floor effects in the adult $\mathrm{CI}$ population using this measure. This task was also chosen to maintain consistency in methods between the present study and our previously completed study involving many of the same participants (Moberly et al, $2018^{15}$ ).

Environmental sound identification was assessed using the FEST-I. The FEST-I is a closed-set, forced-choice test including 25 familiar and easily identifiable environmental sound stimuli. Each sound belongs to one of the five categories identified by Tye-Murray et al (1992) ${ }^{21}$ : (a) human/animal vocalizations and bodily sounds, (b) mechanical sounds, (c) water sounds, (d) aerodynamic sounds, and (e) signaling sounds.

AzBio sentence scores in quiet were included for $22 \mathrm{CI}$ users. These were included when clinically available to investigate for correlations between the measure of QOL subitems and subdomains and a traditional clinical measure of sentence recognition. The eight AzBio lists from the Minimum Speech Test 
Battery, which include 20 sentences spoken by two female and two male talkers, is a widely used measure for adults with CIs.

\section{QOL Measure}

Participants completed the NCIQ (Hinderink et al, 2000 ${ }^{9}$ ), which is a CI-specific QOL measure that includes three domains: physical functioning (including the subdomains of basic sound perception, advanced sound perception, and speech production), psychological functioning (including the subdomain of self-esteem), and social functioning (including the subdomains of activity limitations and social interactions). Ten questions comprise each subdomain, resulting in 60 questions with Likert scale answer categories. Subdomain scores range from 0 (very poor) to 100 (optimal), with the maximum possible overall QOL score of 300. Following Luo et al $(2018)^{13}$, the NCIQ subdomain titles of advanced sound perception (e.g., Are you able to make your voice sound angry, friendly, or sad?) and speech production (e.g., Can you understand strangers without lipreading?) were switched in the present study, and analysis results were interpreted as such.

\section{General Approach}

This study was approved by the local institutional review board. Participants were compensated for their time. Testing was completed in a single session in which participants used their own hearing devices, including hearing aid if typically used. The NCIQ was filled out by the participant after the research visit. Additional AzBio sentence testing in the clinic took approximately 5 minutes, for those participants who had these data.

\section{Data Analyses}

Statistical analyses were performed with SPSS version 25 software (IBM Corporation, Armonk, NY). Group data were analyzed using bivariate correlations of NCIQ scores (total, domain, subdomain, and subitem scores) with clinical and laboratory outcome measures. An a priori power analysis was not completed because previous studies showed weak correlations among objective and QOL measures. Instead, analyses were exploratory in nature. For all measures, an alpha of 0.05 was set. When $p>0.05$, correlations are reported as not significant. Bivariate correlations are shown, with a HolmBonferroni correction applied to correct for multiple comparisons.

\section{Results}

The side of implantation (left, right, or bilateral) did not influence speech recognition scores. Also, no differences in any scores were found for $\mathrm{CI}$ users who wore only $\mathrm{CI}(\mathrm{s})$ versus a CI plus hearing aid. Therefore, the data were collapsed across all participants in subsequent analyses reported in the following paragraphs.

\section{Group Data}

Group mean demographic and audiologic data, screening measures, speech and environmental sound recognition performance, and NCIQ scores are shown in - Table 1. Before performing our main correlation analyses, bivariate correlation analyses were performed among NCIQ total, domain, and subdomain scores with demographic/audiologic factors of participant age, duration of hearing loss (current age minus reported age at the onset of hearing loss), and duration of $\mathrm{Cl}$ use, to determine if these factors should be used as covariates in our main analyses of interest. Results are shown in - Table 2, revealing only one significant correlation (of 27 correlations performed) after Holm-Bonferroni correction. Therefore, none of these factors was treated as a covariate in our main analyses.

Correlations among objective behavioral measures and NCIQ scores are shown in - Table 3. The total NCIQ sum score was not correlated with speech recognition measures after Holm-Bonferroni correction.

\section{Domains and Subdomains}

Correlations between NCIQ domain and subdomain data and outcome measures are also shown in - Table 3. The physical domain was moderately correlated with environmental sound recognition $(r=0.44)$ but was not correlated with other outcome measures after Holm-Bonferroni correction $(r=0.27-0.50)$. The psychological and social domains were not significantly correlated with outcome measures.

Note: Bold values have a significant p value after HolmBonferroni correction. * $\mathrm{p}>0.05 ;{ }^{* *} \mathrm{p}<0.01$

Within the physical domain, the advanced sound perception subdomain showed moderate correlations with CID word recognition and AzBio sentence recognition $(r=0.43$ and 0.48 , respectively). The speech production subdomain (originally labeled by Hinderink et al as the "advanced sound perception" subdomain) was most strongly related to environmental sound recognition $(r=0.56)$.

Within the psychological and social domains, only the correlation between the social interactions subdomain and audiovisual sentence recognition was significant $(r=0.44)$ following Holm-Bonferroni correction.

\section{Subitems}

Results of subitem analyses are shown in -Table 4. Of the 60 individual items on the NCIQ, 27 questions were found to correlate with at least one auditory or audiovisual outcome measure at a $p$ value of $<0.05 ; 14$ questions were significantly correlated after Holm-Bonferroni correction. Negative $r$ values correspond to questions which were recoded in the NCIQ, in which the Likert scale's categories are reversed (e.g., a score of five indicating "never" rather than "always"). CUNY audiovisual word recognition showed the greatest number of correlations with NCIQ, with the magnitude of $r$ values ranging from 0.32 to 0.61 . Three subitems were significantly correlated with all outcome measures after Holm-Bonferroni correction: question 40 ("Can you understand strangers without lipreading?"), question 44 ("Can you make contact easily with other persons despite your hearing problem?"), and question 45 ("Can you hear the difference between a man's voice, a woman's voice, and a child's voice?").

Of the 33 subitems not correlated with any outcome measures, a few similar trends emerged. Many of these 
Table 1 Demographic, Audiologic, Screening, Auditory Outcome, and QOL (NCIQ) Data for Cl Participants

\begin{tabular}{|c|c|c|c|c|}
\hline & Mean & $\mathbf{N}$ & (Min-Max) & SD \\
\hline \multicolumn{5}{|l|}{ Demographics } \\
\hline Age (years) & 66.8 & 44 & $(45-83)$ & 10.1 \\
\hline Socioeconomic status (score) & 26.5 & 44 & $(9-64)$ & 14.5 \\
\hline \multicolumn{5}{|l|}{ Audiologic factors } \\
\hline Age hearing loss began (years) & 26.9 & 44 & $(0-68)$ & 21.1 \\
\hline Duration of deafness (years) & 39.6 & 44 & $(4-76)$ & 19.5 \\
\hline Age at implantation (years) & 59.8 & 44 & $(35-82)$ & 12.6 \\
\hline Duration of $\mathrm{Cl}$ use (years) & 7.0 & 44 & $(1-34)$ & 6.6 \\
\hline Better-ear pure-tone average (dB HL) & 96.3 & 44 & $(22.5-120)$ & 21.5 \\
\hline \multicolumn{5}{|l|}{ Screening measures } \\
\hline Reading (standard score) & 97.6 & 44 & $(77-122)$ & 11.9 \\
\hline Cognitive Mini-Mental State Examination (Tscore) & 53.5 & 43 & $(30-83)$ & 9.9 \\
\hline \multicolumn{5}{|l|}{ Speech/environmental sound recognition } \\
\hline Word recognition (percent correct) & 66.8 & 44 & $(18-98)$ & 21.5 \\
\hline Standard sentence recognition (percent words correct) & 73.4 & 43 & $(12-91)$ & 16.1 \\
\hline AzBio sentence recognition (percent words correct) & 86.5 & 22 & $(40-99)$ & 14.5 \\
\hline High-variability sentence recognition (percent words correct) & 57.2 & 43 & $(7-97)$ & 23.7 \\
\hline Audiovisual sentence recognition (percent words correct) & 90.5 & 44 & $(52-100)$ & 11.7 \\
\hline Environmental sound recognition (percent correct) & 60.9 & 43 & $(16-88)$ & 14.8 \\
\hline \multicolumn{5}{|l|}{ QOL } \\
\hline NCIQ (sum score) & 204.4 & 43 & $(116-269)$ & 39.0 \\
\hline Physical-basic sound perception & 62.6 & 44 & $(25-100)$ & 18.4 \\
\hline Physical-advanced sound perception & 59.6 & 44 & $(28-98)$ & 17.7 \\
\hline Physical-speech production & 82.3 & 44 & $(28-100)$ & 15.1 \\
\hline Physical (sum score) & 68.2 & 44 & $(32-99)$ & 14.4 \\
\hline Psychological-self-esteem (sum score) & 66.4 & 43 & $(19-92)$ & 16.9 \\
\hline Social-activity limitations & 77.2 & 43 & $(18-98)$ & 15.4 \\
\hline Social-social interactions & 63.4 & 43 & $(19-83)$ & 12.5 \\
\hline Social (sum score) & 70.3 & 43 & $(18-90)$ & 13.4 \\
\hline
\end{tabular}

Table 2 Bivariate Correlations among QOL Measures from the NCIQ and Demographic/Audiologic Factors for CI Participants

\begin{tabular}{|l|l|l|l|}
\hline$r$ Values & Age (Years) & Duration of Hearing Loss (Years) & Duration of CI Use (Years) \\
\hline NCIQ (sum score) & -0.25 & 0.14 & 0.17 \\
\hline Physical-basic sound perception & -0.08 & 0.14 & -0.09 \\
\hline Physical-advanced sound perception & $-0.39^{*}$ & 0.04 & 0.10 \\
\hline Physical-speech production & $-0.47^{* *}$ & -0.05 & 0.22 \\
\hline Physical (sum score) & $-0.37^{*}$ & 0.06 & 0.08 \\
\hline Psychological-self-esteem (sum score) & -0.13 & 0.07 & 0.11 \\
\hline Social-activity limitations & -0.08 & 0.25 & 0.30 \\
\hline Social-social interactions & -0.21 & 0.30 & 0.12 \\
\hline Social (sum score) & -0.14 & 0.28 & 0.23 \\
\hline
\end{tabular}

Note: Bold values have a significant $p$ value after Holm-Bonferroni corr. 
Table 3 Bivariate Correlations among QOL Measures from the NCIQ and Speech and Environmental Sound Outcome Measures for Cl Participants

\begin{tabular}{|c|l|l|l|l|l|l|}
\hline$r$ Values & $\begin{array}{l}\text { Word } \\
\text { Recognition } \\
\text { (Percent } \\
\text { Correct) }\end{array}$ & $\begin{array}{l}\text { Standard } \\
\text { Sentence } \\
\text { Recognition } \\
\text { (Percent } \\
\text { Correct } \\
\text { Words) }\end{array}$ & $\begin{array}{l}\text { AzBio } \\
\text { Sentence } \\
\text { Recognition } \\
\text { (Percent } \\
\text { Correct } \\
\text { Words) }\end{array}$ & $\begin{array}{l}\text { High-Variability } \\
\text { Sentence } \\
\text { Recognition } \\
\text { (Percent } \\
\text { Correct Words) }\end{array}$ & $\begin{array}{l}\text { Audiovisual } \\
\text { Sentence } \\
\text { Recognition } \\
\text { (Percent } \\
\text { Correct } \\
\text { Words) }\end{array}$ & $\begin{array}{l}\text { Environmental } \\
\text { Sound Recognition } \\
\text { (Percent Correct) }\end{array}$ \\
\hline NCIQ (sum score) & 0.21 & 0.22 & 0.22 & 0.23 & $0.41^{*}$ & 0.31 \\
\hline $\begin{array}{l}\text { Physical-basic } \\
\text { sound perception }\end{array}$ & 0.08 & 0.07 & 0.34 & 0.04 & 0.15 & 0.15 \\
\hline $\begin{array}{l}\text { Physical-advanced } \\
\text { sound perception }\end{array}$ & $0.43^{* *}$ & $0.36^{*}$ & $0.48^{*}$ & $0.37^{*}$ & $0.36^{*}$ & $0.40^{*}$ \\
\hline Physical-speech production & $0.36^{*}$ & $0.35^{*}$ & $0.49^{*}$ & 0.27 & $0.38^{*}$ & $0.56^{* *}$ \\
\hline Physical (sum score) & $0.35^{*}$ & 0.30 & $0.50^{*}$ & 0.27 & $0.35^{*}$ & $0.44^{* *}$ \\
\hline $\begin{array}{c}\text { Psychological-self-esteem } \\
\text { (sum score) }\end{array}$ & 0.16 & 0.19 & 0.08 & 0.17 & $0.39^{*}$ & 0.26 \\
\hline Social-activity limitations & 0.02 & 0.10 & 0.07 & 0.17 & 0.26 & 0.15 \\
\hline Social-social interactions & 0.10 & 0.14 & 0.07 & 0.19 & $0.44^{* *}$ & 0.17 \\
\hline Social (sum score) & 0.06 & 0.12 & 0.07 & 0.18 & $0.36^{*}$ & 0.16 \\
\hline
\end{tabular}

Note: Bold values have a significant $p$ value after Holm-Bonferroni correction. ${ }^{*} p<0.05$.

${ }^{*} p<0.01$.

NCIQ questions were labeled as "physical-basic sound perception" and "psychological-self-esteem" subdomains, indicating that our functional outcome measures may not provide a complete picture of an individual's perceived performance with $\mathrm{CI}$.

\section{Discussion}

The purpose of this study was to determine how patientreported QOL measured by the NCIQ's domains, subdomains, and subitems relates to objective outcome measures in adult CI users, and what this relationship can uncover about presently used clinical measures.

First, we hypothesized that the domains, subdomains, and subitems of the NCIQ would demonstrate stronger correlations with objective performance measures than the NCIQ total score. Consistent with previous studies, the total NCIQ score did not correlate with auditory measures and may not best capture $\mathrm{CI}$

Second, we predicted that the auditory measures used in this study would demonstrate stronger correlations with NCIQ scores than traditional clinical measures. Relations between auditory outcome measures and subdomain QOL scores were stronger than those in previous work (Olze et al, $2012^{18}$; Capretta and Moberly, 2016 ${ }^{2}$; Moberly et al, 2018 ${ }^{15}$ ). This finding may be a result of the incorporation of speech recognition tests that could be considered more "real life" by providing greater talker variabiity and greater linguistic complexity that relate better to real-world communication environments. Moreover, the larger sample size in this study also powered these analyses more effectively than some previous studies. Thus, the new and varied outcome measures likely tapped into the group's auditory abilities in a diverse way.
The addition of the CUNY audiovisual sentence recognition task added a new dimension to the relation of outcome measures and QOL. The CUNY task correlated with all domain scores and all subdomains (except for social-activity limitations). This task highlights a CI user's ability to understand audiovisual conversational speech, a crucial skill which is not evaluated in traditional clinical measures. It is not unexpected, then, that this measure is significantly correlated with the social domain of the NCIQ (example question: "Are you left aside in company because of your hearing impairment?") and with the overall QOL. This finding is also highly relevant considering that combined audiovisual conversation is the most common method of communication among adult $\mathrm{CI}$ users (Dorman et al, 2016 ${ }^{3}$ ).

Clinical sentence recognition ability, measured by AzBio, is a standard measure that was included in analyses to determine the relation of traditional clinical outcome measures to QOL domain, subdomain, and subitem scores. Although previous work has identified low-to-moderate correlations between AzBio scores and total NCIQ score, our group was interested in examining how this measure might highlight certain selfreported hearing abilities addressed in the NCIQ subitems. Consistent with past studies, the present study indicated that AzBio scores were not correlated with the total NCIQ score; however, ten of the subitems were correlated with the AzBio score, with three of these items showing strong correlations $(r=0.66-0.70)$. Traditional outcome measures have not shown strong correlations with NCIQ total score and domains, but may provide valuable insight into particular hearing-related struggles for counseling and programming purposes. For example, particular subitems related to the AzBio score included discriminating men's, women's, and children's voices (question $45 ; r=0.70$ ), and speech production of various emotions 


\begin{tabular}{|c|c|c|c|c|c|c|c|c|c|c|c|c|c|c|}
\hline 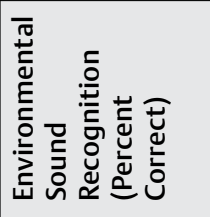 & $\begin{array}{l}\text { L } \\
0\end{array}$ & $\begin{array}{c}\tilde{N} \\
\tilde{O} \\
1\end{array}$ & $\stackrel{m}{\circ}$ & 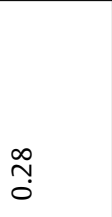 & 苂 & $\frac{0}{\stackrel{0}{i}}$ & $\stackrel{*}{\leftarrow}$ & 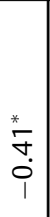 & $\begin{array}{l}\stackrel{9}{2} \\
\text { O̦ } \\
1\end{array}$ & $\frac{0}{0}$ & $\begin{array}{l}\text { શ̂} \\
\text { ì } \\
1\end{array}$ & $\stackrel{*}{\stackrel{*}{\sim}}$ & $\bar{m}$ & $\begin{array}{l}\stackrel{0}{1} \\
\stackrel{0}{i}\end{array}$ \\
\hline 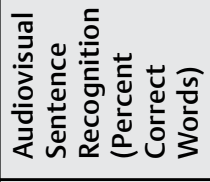 & 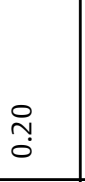 & 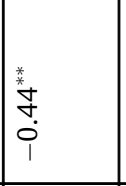 & 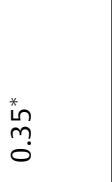 & $\stackrel{n}{0}$ & $\bar{m}$ & 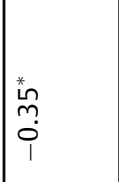 & m. & $\mid \begin{array}{l}* \\
\dot{0} \\
+ \\
0 \\
1 \\
1\end{array}$ & 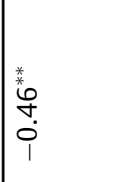 & 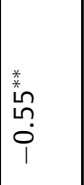 & 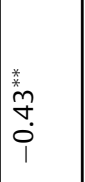 & 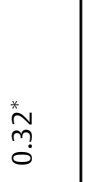 & $\frac{9}{0}$ & 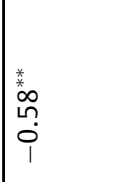 \\
\hline 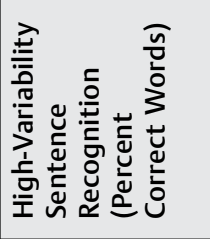 & $\begin{array}{l}\tilde{O} \\
0 \\
0\end{array}$ & $\mid \begin{array}{c}\hat{N} \\
0 \\
i\end{array}$ & $\overline{0}$ & $\stackrel{\text { Hे }}{m}$ & $\stackrel{\sim}{\circ}$ & $\mid$\begin{tabular}{l}
0 \\
\multirow{2}{0}{} \\
$i$ \\
1
\end{tabular} & 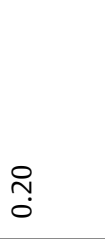 & $\begin{array}{c}m \\
m \\
0 \\
1\end{array}$ & 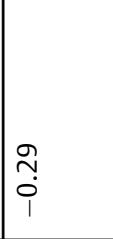 & Е. & $\begin{array}{l}\stackrel{*}{*} \\
\stackrel{0}{0} \\
1\end{array}$ & $\begin{array}{l}\text { సे } \\
\text { Oे }\end{array}$ & 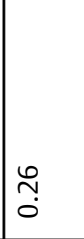 & $\begin{array}{l}\infty \\
\stackrel{\infty}{N} \\
\stackrel{0}{1} \\
1\end{array}$ \\
\hline 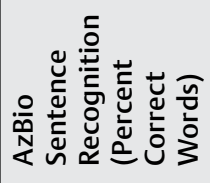 & $\begin{array}{l}\text { 免 } \\
\stackrel{0}{0}\end{array}$ & $\bar{i}$ & $\stackrel{\sim}{\check{0}}$ & $\stackrel{q}{q}$ & 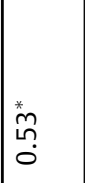 & $\frac{0}{i}$ & $\stackrel{1}{0}$ & $\begin{array}{l}\stackrel{n}{\cong} \\
0 \\
1\end{array}$ & 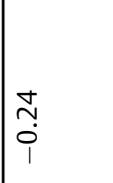 & $\stackrel{8}{0}$ & 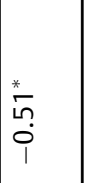 & 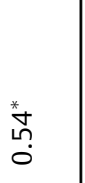 & mo & $\frac{0}{0}$ \\
\hline 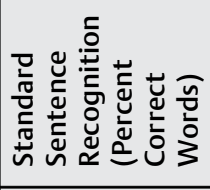 & 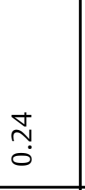 & $\begin{array}{l}0 \\
\\
1 \\
\end{array}$ & స̦ & $\begin{array}{l}\text { o } \\
\stackrel{m}{0} \\
0\end{array}$ & $\stackrel{m}{\circ}$ & $\begin{array}{l}\stackrel{m}{N} \\
\stackrel{i}{i} \\
\end{array}$ & Nָ & $\begin{array}{c}* \\
m \\
m \\
0 \\
1 \\
\end{array}$ & $\begin{array}{l}\bar{m} \\
\vdots \\
i\end{array}$ & 旾 & $\begin{array}{l}0 \\
0 \\
m \\
i \\
i\end{array}$ & m. & $\begin{array}{l}\tilde{N} \\
0\end{array}$ & $\begin{array}{l}\text { * } \\
\text { m. } \\
0 \\
i \\
1\end{array}$ \\
\hline 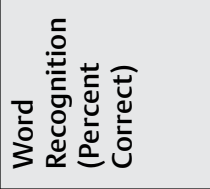 & $\frac{\sigma}{0}$ & $\frac{n}{\operatorname{c}}$ & $\stackrel{\infty}{\circ} \stackrel{0}{0}$ & $\stackrel{\stackrel{\sim}{\sim}}{\stackrel{0}{0}}$ & 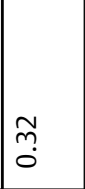 & $\tilde{\sigma}$ & $\frac{\sigma}{0}$ & $\begin{array}{c}\bar{m} \\
\dot{0} \\
1\end{array}$ & 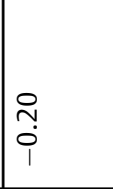 & $\begin{array}{l}\text { ô } \\
0 \\
0\end{array}$ & 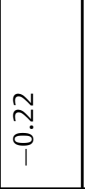 & $\stackrel{\stackrel{L}{N}}{\stackrel{0}{0}}$ & $\begin{array}{l}\text { 粚 } \\
\stackrel{5}{0}\end{array}$ & $\begin{array}{l}\stackrel{n}{N} \\
\stackrel{1}{1} \\
1\end{array}$ \\
\hline 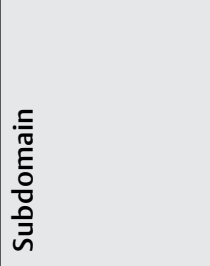 & 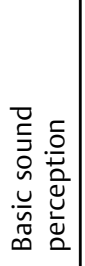 & 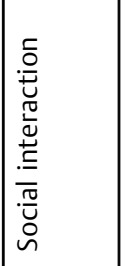 & 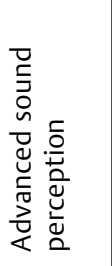 & 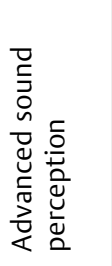 & 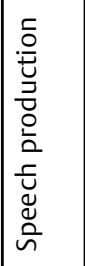 & 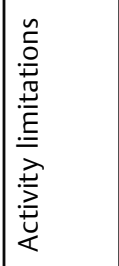 & 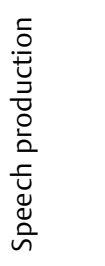 & 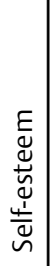 & 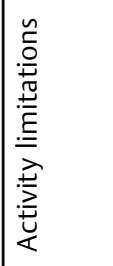 & 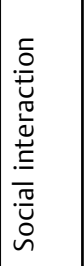 & 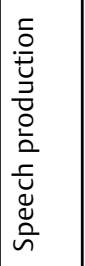 & 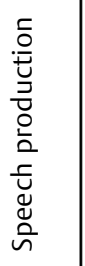 & 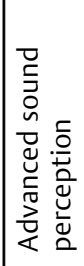 & 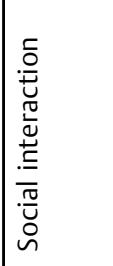 \\
\hline 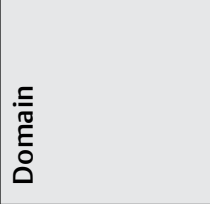 & $\begin{array}{l}\bar{v} \\
\stackrel{\sqrt{n}}{a} \\
\text { a }\end{array}$ & \begin{tabular}{|l}
$\bar{\pi}$ \\
$\bar{\complement}$ \\
$\stackrel{0}{\circ}$
\end{tabular} & 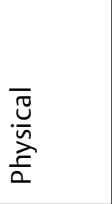 & $\begin{array}{l}\overline{\frac{v}{n}} \\
\stackrel{\underline{a}}{a}\end{array}$ & 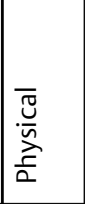 & $\mid \begin{array}{l}\bar{\pi} \\
\stackrel{\bar{U}}{\breve{~}} \\
\backsim\end{array}$ & $\begin{array}{l}\overline{\sqrt{n}} \\
\stackrel{\underline{a}}{a} \\
\text { a }\end{array}$ & 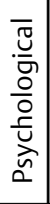 & 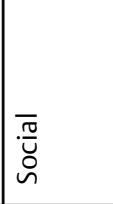 & 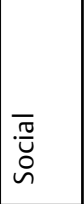 & 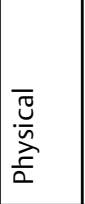 & $\begin{array}{l}\overline{\frac{\pi}{n}} \\
\stackrel{\underline{n}}{a} \\
\text { a }\end{array}$ & 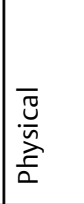 & 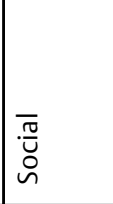 \\
\hline 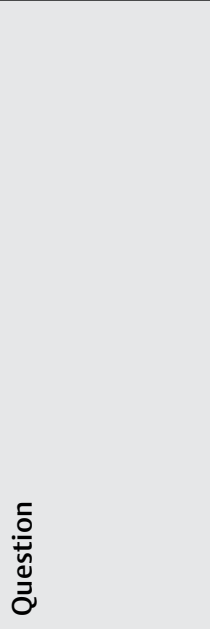 & 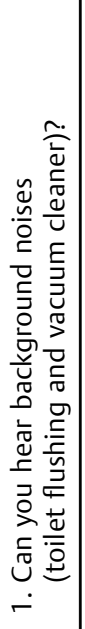 & 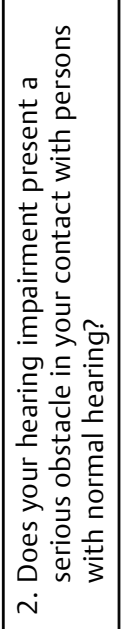 & 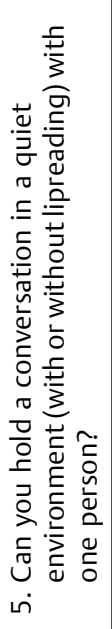 & 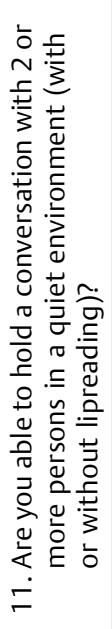 & 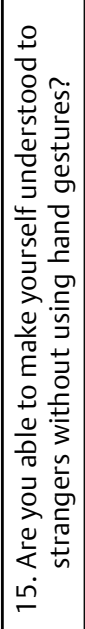 & 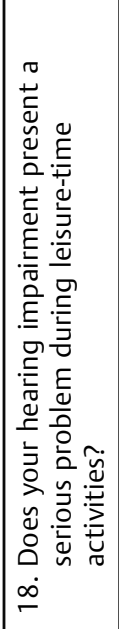 & 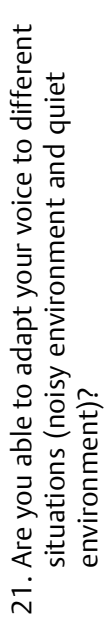 & 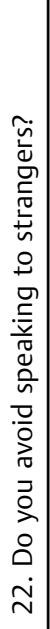 & 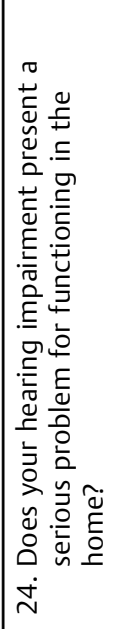 & 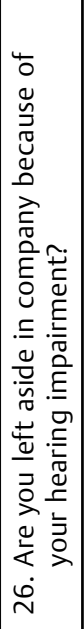 & 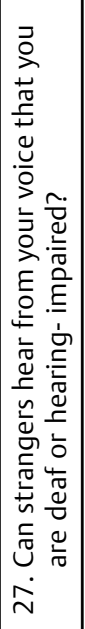 & 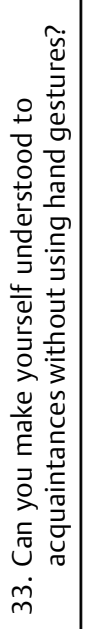 & 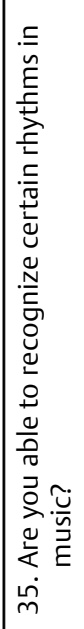 & 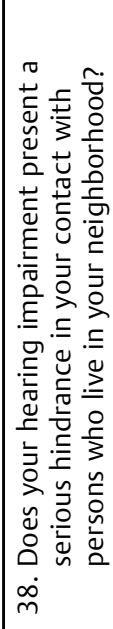 \\
\hline
\end{tabular}




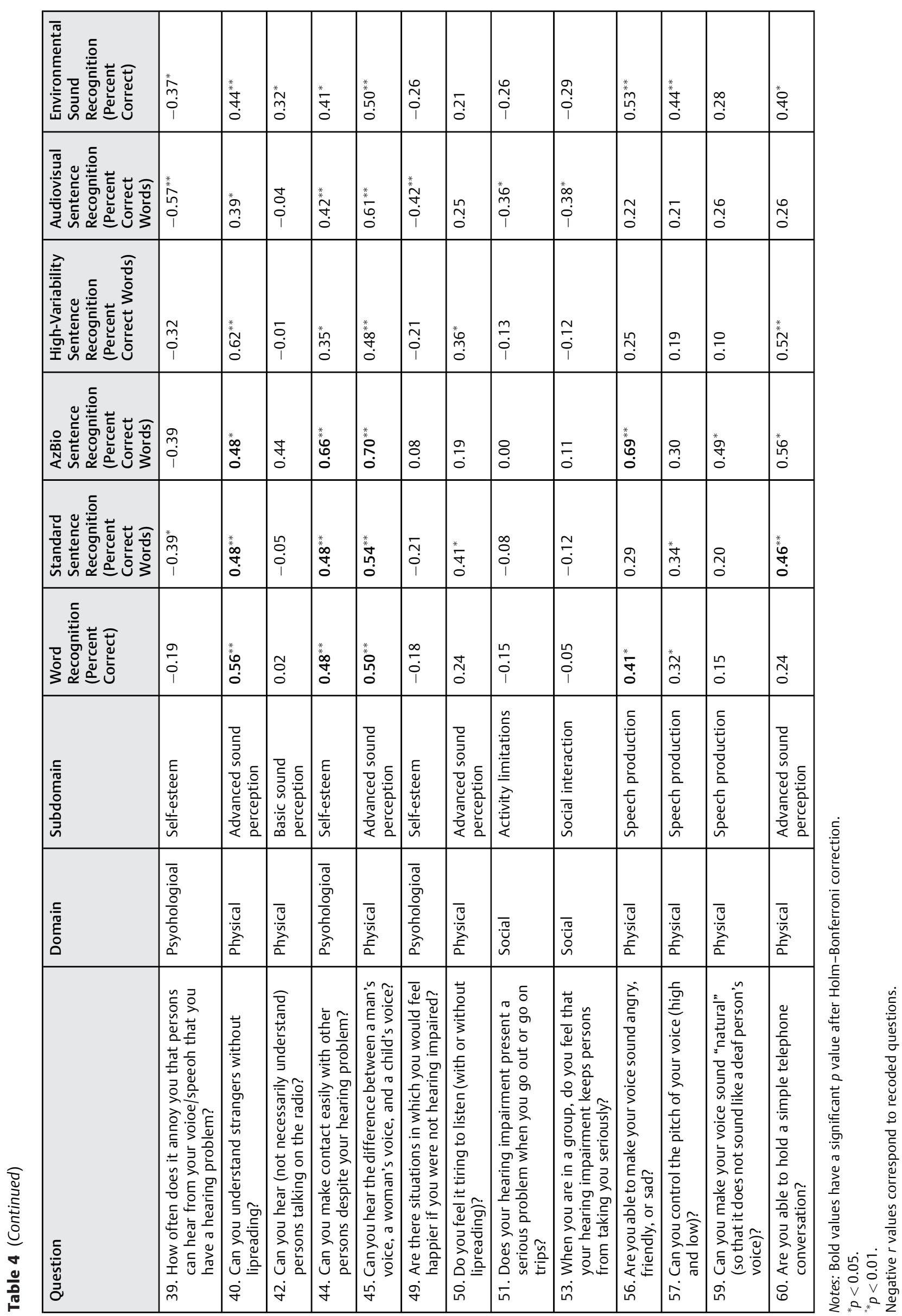


(question $56 ; r=0.69$ ). Unfortunately, only 22 of the $44 \mathrm{CI}$ users in this study had clinical AzBio scores available for analyses; a larger sample may have indicated other relating subdomains and subitems within the NCIQ.

Environmental sound recognition, as measured by the FEST-I, is another nontraditional outcome measure that has been found to relate to the overall speech recognition ability as well as to the total NCIQ score (Capretta and Moberly, $2016^{2}$ ). When examining the relationship between FEST-I and the various domains, subdomains, and subitems of the NCIQ, three correlations in particular were identified. First, the FEST-I was moderately correlated with the speech production subscale, which examines volume and pitch control, stigma, and vocal expression of emotions. As environmental sound identification relies strongly on a CI user's pitch perception, it is possible that this skill is a mechanism behind the task's correlation with this subscale. This was also seen in the original development and validation study by Hinderink et al $(2000)^{9}$, in which their Environmental Sounds Identification Test showed a moderate correlation $(r=0.59)$ with speech production. In this study, moderate correlation was seen between FEST-I and the advanced sound perception subdomain and, unsurprisingly, the physical domain, which encompasses the basic sound perception, advanced sound perception, and speech production subdomains.

Fourteen of the 60 individual subitems of the NCIQ were associated with at least one outcome measure after HolmBonferroni correction. Most notable were question 40 ("Can you understand strangers without lipreading?"), question 44 ("Can you make contact easily with other persons despite your hearing problem?"), and question 45 ("Can you hear the difference between a man's voice, a woman's voice, and a child's voice?"), which correlated with all outcome measures. These three questions touch on conversational skills, selfesteem, and social interaction in a way that is not traditionally tested directly in clinical measures. Although clinical speech recognition tests may include the voices of men, women, and children, they are not evaluated for accuracy in identifying the talker type. These tasks are also traditionally presented in an auditory-only format, reducing the ability of the $\mathrm{CI}$ user to rely on visual cues. These highly correlated NCIQ subitems could plausibly be used to generate an abridged NCIQ and to encourage conversation and counseling between clinicians and patients. For example, a low self-reported rating on question 60, "Are you able to hold a simple telephone conversation?" which is related to standard and high-variability sentence recognition (e.g., unfamiliar callers) and environmental sound recognition (e.g., phone ringing or a busy signal, both stimuli included in this task), could lead to counseling on telephone practice or an evaluation for assistive devices such as a captioned phone.

\section{Limitations}

By examining responses on the NCIQ in this more detailed fashion, we were able to find which domains, subdomains, and subitems were "high-yield" in the adult CI population, at least in terms of their relationship with objective behavioral measures. However, a noteworthy consideration and weakness of this study is that, aside from AzBio, the outcome measures used in this study are not traditional clinical measures and did not include testing in noise, which is arguably a relevant measure to CI user's everyday performance.

An additional weakness of the present study is that it is limited to experienced $\mathrm{CI}$ users. It will be worth exploring whether similar relationships between objective and QOL measures exist for $\mathrm{CI}$ candidates who are evaluated before and then again after implantation. We predict that both outcome measures and NCIQ scores will increase from baseline after implantation, but it is also plausible that the relationship between outcome measures and the various domains, subdomains, and subitems ofthe NCIQ may progress in new $\mathrm{CI}$ users in a fashion that is distinct from findings in experienced CI users. Other patient populations of interest to examine in future studies include adult prelingually deafened CI users and pediatric or adolescent CI users, whose ultimate subjective QOL outcomes may depend differentially on physical, psychological, and/or social domains. Last, because this study was exploratory in nature, an a priori power analysis was not performed; thus, it is possible that our study was underpowered to identify some true correlations among measures.

\section{Conclusion}

The present study examined the relationship between functional measures of speech and environmental sound recognition and patient-reported QOL with CI. Our findings can be interpreted in two ways: first, traditional outcome measures may need expansion to include additional skills, such as audiovisual speech and environmental sound recognition, because these were found to be related to subjective outcomes. In addition, to this group's knowledge, the individual subitems of the NCIQ have not been examined in relation to other traditional outcome measures, such as Hearing in Noise Test (HINT), Consonant-Nucleus-Consonant Test (CNC), or Bamford-Kowal-Bench Speech in Noise Test (BKB-SIN). It is quite possible that, although significant relations between these measures and NCIQ domains/subdomains have not been observed, certain questions might relate.

Findings indicate that certain domains, subdomains, and subitems of the NCIQ are correlated with several outcome measures, indicating that this questionnaire does valuably capture many of the everyday communication difficulties that CI users face. By contrast, some of these listening situations are not assessed in traditional clinical outcome measures; therefore, the data captured by the NCIQ may prove to be useful for providing various forms of auditory rehabilitation to the $\mathrm{CI}$ user.

\section{Abbreviations}

CI cochlear implant

CID Central Institute for the Deaf

FEST-I Familiar Environmental Sound Test

NCIQ Nijmegen Cochlear Implant Questionnaire

PRO patient-reported outcome

QOL quality of life

SD standard deviation 


\section{Notes}

DataData from this manuscript were presented at the annual conference of the American Academy of Audiology, Columbus, OH, March 27-30, 2019.

\section{Funding}

This work was supported by the American Otological Society Clinician-Scientist Award and the National Institutes of Health, National Institute on Deafness, and Other Communication Disorders (NIDCD) Career Development Award 5K23DC015539-02 to Aaron Moberly. Research reported in this article received IRB approval from the Ohio State University. ACM receives grant funding support from Cochlear Americas for an unrelated investigatorinitiated research study.

\section{References}

1 Boothroyd A, Hanin L, Hnath T. CUNY Laser Videodisk of Everyday Sentences. New York, NY: Speech and Hearing Sciences Research Center, City University of New York; 1985

2 Capretta NR, Moberly AC. Does quality of life depend on speech recognition performance for adult cochlear implant users? Laryngoscope 2016;126:699-706

3 Dorman MF, Liss J, Wang S, Berisha V, Ludwig C, Natale SC. Experiments on auditory-visual perception of sentences by users of unilateral, bimodal, and bilateral cochlear implants. J Speech Lang Hear Res 2016;59:1505-1519

4 FDA-NIH Biomarker Working Group. BEST (Biomarkers, EndpointS, and other Tools) Resource [Internet]. Co-published by Food and Drug Administration, Silver Spring, MD \& National Institutes of Health, Bethesda, MD. 2016

5 Folstein MF, Folstein SE, McHugh PR. "Mini-mental state": a practical method for grading the cognitive state of patients for the clinician. J Psychiatr Res 1975;12:189-198

6 Gatehouse S, Noble W. The speech, spatial, and qualities of hearing scale (SSQ). Int J Audiol 2004;43:85-99

7 Gaylor JM, Raman G, Chung M, Lee J, Rao M, Lau J, Poe DS. Cochlear implantation in adults: a systematic review and meta-analysis. J Otolaryngol Head Neck Surg 2013;139:265-272

8 Gilbert JL, Tamati TN, Pisoni DB. Development, reliability, and validity of PRESTO: a new high-variability sentence recognition test. J Am Acad Audiol 2013;24:26-36
9 Hinderink JB, Krabbe PF, Van Den Broek P. Development and application of a health-related quality-of-life instrument for adults with cochlear implants: the Njimegen Cochlear Implant Questionnaire. J Otolaryngol Head Neck Surg 2000; 123:756-765

10 Hirsh I, Davis H, Silverman S, Reynolds E, Eldert E, Benson R. Development of materials for speech audiometry. J Speech Hear Disord 1952;17:321-337

11 IEEE. IEEE recommended practice for speech quality measurements. IEEE Report, 297. The Institute of Electrical and Electronics Engineers, Inc.New York1969

12 Krabbe PF, Hinderink JB, Van Den Broek P. The effect of cochlear implant use in postlingually deaf adults. Int J Technol Assess Health Care 2000;16:864-873

13 Luo X, Kern A, Pulling KR. Vocal emotion recognition performance predicts the quality of life in adult cochlear implant users. J Acoust Soc Am 2018;144:429-435

14 McRackan T, Bauschard M, Hatch JL, Franko-Tobin E, Droghini R, Nguyen SA, Dubno JR. Meta-analysis of quality-of-life improvement after cochlear implantation and associations with speech recognition abilities. Laryngoscope 2018;128:982-990

15 Moberly AC, Harris MS, Boyce L, Vasil K, Wucinich T, Pisoni DB, Baxter J, Ray C, Shafiro V. Relating quality of life to outcomes and predictors in adult cochlear implant users: are we measuring the right things? Laryngoscope 2018;128:959-966

16 Moberly AC, Vasil KJ, Ray C. Visual reliance during speech recognition in cochlear implant users and candidates. J Am Acad Audiol 2019 [Epub ahead of print]

17 Newman CW, Weinstein BE, Jacobson GP, Hug GA. The Hearing Handicap Inventory for Adults: psychometric adequacy and audiometric correlates. Ear Hear 1990;11:430-433

18 Olze H, Grabel S, Haupt H, Forster U, Mazurek B. Extra benefit of a second cochlear implant with respect to health-related quality of life and tinnitus. Otol Neurotol 2012;33:1169-1175

19 Shafiro V. Development of a large-item environmental sound test and the effects of short-term training with spectrally-degraded stimuli. Ear Hear 2008;29:775-790

20 Spahr AJ, Dorman MF, Litvak LM, Van Wie S, Gifford RH, Loizou PC, Loiselle LM, Oakes T, Cook S. Development and validation of the AzBio sentence lists. Ear Hear 2012;33:112-117

21 Tye-Murray N, Tyler RS, Woodworth GG, Gantz BJ. Performance over time with a nucleus or ineraid cochlear implant. Ear Hear 1992;13:200-209

22 Wilkinson GS, Robertson GJ. 2006Wide Range Achievement Test4 (WRAT-4). Lutz, FL: Psychological Assessment Resources, Inc. 